\title{
Infinite-Period Density-Matrix Model for Terahertz-Frequency Quantum Cascade Lasers
}

\author{
Aleksandar Demić, Andrew Grier, Zoran Ikonić, Alexander Valavanis, Craig A. Evans, Reshma Mohandas, \\ Lianhe Li, Edmund H. Linfield, Alexander Giles Davies, and Dragan Indjin
}

\begin{abstract}
In this work, we present a density-matrix model, which considers an infinite quantum cascade laser (QCL) and models transport via a nearest neighbor approximation. We will discuss derivation of output parameters of the model in detail and show the direct mathematical link to the semiclassical rate equation approach. This model can be extended to an arbitrary number of states in the QCL period, without a priori specification of upper and lower lasing level. Application of the model to various QCL structures is possible, including bound-to-continuum structures, which typically employ a large number of states per period. The model has been applied to a 2-THz bound-to-continuum QCL, and a very good agreement with measured $V-I$ characteristics is obtained along with qualitative agreement with measured $L-I$ characteristics in terms of dynamic range.
\end{abstract}

Index Terms-Submillimeter wave technology, quantum cascade lasers (QCLs).

\section{INTRODUCTION}

$\mathbf{T}$ ERAHERTZ-FREQUENCY quantum cascade lasers (THz QCLs) are powerful semiconductor sources of coherent radiation in the $\mathrm{THz}$ band [1] with potential applications in free-space communications, medical diagnostics, and chemical sensing [2]-[7].

The lasing frequency of a THz QCL is dominated by the separation between a pair of energy states within a periodic semiconductor heterostructure (typically GaAs/AlGaAs), and by engineering the thickness of layers in the active region, it is possible to generate a wide range of operating frequencies (1.2-5.6 THz [8]-[10]). However, the required energy difference between the lasing states is very small $(\sim 10 \mathrm{meV})$, and thermal

Manuscript received February 10, 2017; revised April 4, 2017; accepted May 1, 2017. Date of publication June 23, 2017; date of current version June 29 , 2017. This work was supported by the NATO Science for Peace program (SfP 984068 grant), by the European Cooperation in Science and Technology (COST Action BM1205), by the Engineering and Physical Science Research Council U.K. (EPSRC-UK COTS grant EP/J017671/1), and by the Royal Society (Wolfson Research Merit Award WM150029). Data associated with this work are available from the Research Data Leeds repository under a CC-BY license at: https://doi.org/10.5518/194. (Corresponding author: Aleksandar Demić.)

A. Demić, A. Grier, Z. Ikonić, A. Valavanis, C. A. Evans, R. Mohandas, L. Li, E. H. Linfield, A. G. Davies, and D. Indjin are with the School of Electronic and Electrical Engineering, University of Leeds, Leeds LS2 9JT, U.K. (e-mail: elade@leeds.ac.uk; el09a2g@leeds.ac.uk; Z.Ikonic@leeds.ac.uk; a.valavanis@leeds.ac.uk; C.A.Evans@leeds.ac.uk; R.AnamariMohandas@ leeds.ac.uk; L.H.Li@leeds.ac.uk; e.h.linfield@leeds.ac.uk; G.Davies@leeds. ac.uk; D.Indjin@leeds.ac.uk).

Color versions of one or more of the figures in this paper are available online at http://ieeexplore.ieee.org.

Digital Object Identifier 10.1109/TTHZ.2017.2705481 excitation of carriers can rapidly degrade device performance. THz QCLs currently operate at cryogenic temperatures (below $200 \mathrm{~K}$ [11] in pulsed mode and typically to few tens of Kelvin in continuous-wave $(\mathrm{CW})$ operation, without an external magnetic field).

Various models for transport in QCLs exist [12]: most commonly employing semiclassical approaches such as selfconsistent rate-equation (RE) modeling, which considers nonradiative transitions of carriers due to the various scattering mechanisms, including interactions of electrons with longitudinal optical (LO) and acoustic phonons (AP), alloy disorder (AD), interface roughness (IFR), ionized impurities (II) and other electrons (CC). These models are semiclassical because they consider transitions of discrete electrons between energy levels and neglect coherence effects and quantum mechanical dephasing. Although RE models are usually computationally efficient and provide insight into the scattering behavior, they are unable to correctly describe transport between adjacent periods of a QCL structure [13] because they do not take injection barrier thickness into account in transport calculations. This leads to the prediction of instantaneous transport between the periods, whereas the actual transport that occurs is based on the resonant tunneling.

Alternative approaches based on density-matrix (DM) modeling include quantum transport effects and are able to overcome known shortcomings of RE models, while keeping reasonable computational complexity when compared to more extensive quantum mechanical models such as nonequilibrium Green's function approaches [14]-[18]. DM models are frequently applied to simplified QCL models, containing just two or three states per period [19]-[24]. Output of such models is usually combined with RE output [12], [22] through addition of the coherent correction term obtained by analytical analysis for the lasing transition. Although this approach reduces the computational complexity, it results in a cumbersome set of analytic expressions, which is inconvenient for bound-to-continuum (BTC) THz QCLs [25], since these have a large number of states per module.

In this work, we develop an improved general DM approach that extends the model presented in [26], applicable for an arbitrary number of states per module. The model does not require a priori knowledge on which states dominate the electron transport. The DM method has been proved successful for a variety of 
QCL simulations, including quantum dot QCLs [27], nonlinear effects [28], and self-mixing-effect-based interferometry [29].

In Section II, we describe the numerical implementation of a DM formalism for an idealized QCL structure with an infinite number of periods and provide a theoretical discussion of the derivation of the output parameters. In Section III, we give a comparison of the RE and the DM model application to a $\sim 2-\mathrm{THz}$ BTC QCL structure.

\section{THEORETICAL MODEL}

The previously published extended-DM model [26] considers three adjacent periods of the QCL structure. The Hamiltonian for this structure can be represented as a $3 \times 3$ block matrix

$$
H=\left(\begin{array}{lll}
H_{U U} & H_{U C} & H_{U D} \\
H_{C U} & H_{C C} & H_{C D} \\
H_{D U} & H_{D C} & H_{D D}
\end{array}\right)
$$

in which the block subscripts describe interaction terms between the central (C), upper (U), and lower (D) periods. The off-diagonal elements correspond to the coupling between the periods labeled by the corresponding indices. Every block in (1) has the size of $N \times N$, where $N$ is the number of states in a single module. We employ wave functions from a single period as the basis for the density matrix operator, and the corresponding density matrix will have similar form to the Hamiltonian in (1). This would generally result in nine block equations; however, we can greatly simplify the problem if we neglect direct coupling between the "U" and "D" modules, $\mathrm{H}_{\mathrm{UD}}=\mathrm{H}_{\mathrm{DU}}=0$, and use the symmetry of the structure: $\mathrm{H}_{\mathrm{CD}}=\mathrm{H}_{\mathrm{UC}}$ and $\mathrm{H}_{\mathrm{DC}}=\mathrm{H}_{\mathrm{CU}}$, $\mathrm{H}_{\mathrm{UU}}=\mathrm{H}_{\mathrm{CC}}+e K L_{\mathrm{P}}$, and $\mathrm{H}_{\mathrm{DD}}=\mathrm{H}_{\mathrm{CC}}-e K L_{\mathrm{P}}$, where $K$ is the applied external electric field and $L_{\mathrm{P}}$ is the length of a single module of the structure. The model then becomes identical to the one in [26]. It is also convenient to switch to numerical indices $\mathrm{H}_{\mathrm{CC}}=\mathrm{H}_{1}, \mathrm{H}_{\mathrm{UC}}=\mathrm{H}_{2}$, and $\mathrm{H}_{\mathrm{CU}}=\mathrm{H}_{3}$. The Hamiltonian and the corresponding density matrix now, respectively, have the forms:

$$
\mathrm{H}=\left(\begin{array}{ccc}
\mathrm{H}_{1}+e K L_{\mathrm{P}} & \mathrm{H}_{2} & 0 \\
\mathrm{H}_{3} & \mathrm{H}_{1} & \mathrm{H}_{2} \\
0 & \mathrm{H}_{3} & \mathrm{H}_{1}-e K L_{\mathrm{P}}
\end{array}\right)
$$

and

$$
\rho=\left(\begin{array}{ccc}
\rho_{1} & \rho_{2} & 0 \\
\rho_{3} & \rho_{1} & \rho_{2} \\
0 & \rho_{3} & \rho_{1}
\end{array}\right)
$$

The Hamiltonian block $\mathrm{H}_{1}$ describes the central period and is composed of tight-binding energies (on the main diagonal) and optical coupling terms of the form $H_{i j}=e z_{i j} A_{\text {inc }}$, where $z_{i j}$ are dipole matrix elements, and $A_{\text {inc }}$ is the electric field of the incident light. Depending on the form of $A_{\text {inc }}$, it is possible to simplify the model further. In this work, we use the nonrotating-wave approximation (NRWA) presented in [26] and assume that optical field has the form $A_{\text {inc }}=A_{0}\left(\mathrm{e}^{\mathrm{i} \omega t}+\mathrm{e}^{-\mathrm{i} \omega t}\right)$. The Hamiltonian block of the central period can, therefore, be presented as $\mathrm{H}_{1}=\mathrm{H}^{\mathrm{dc}}+\mathrm{H}^{\mathrm{ac}+} \mathrm{e}^{\mathrm{i} \omega t}+\mathrm{H}^{\mathrm{ac}-} \mathrm{e}^{-\mathrm{i} \omega t}$, where $\mathrm{H}^{\mathrm{dc}}$ refers to the main diagonal of $\mathrm{H}_{1}$, while the remaining ac terms have equal amplitudes $\left(\left|\mathrm{H}^{\mathrm{ac}+}\right|=\left|\mathrm{H}^{\mathrm{ac}-}\right|\right)$ and will be labeled as $\mathrm{H}^{\text {ac }}$ (where $H_{i j}^{\mathrm{ac}}=e z_{i j} A_{0}, i \neq j$ ). Hamiltonian blocks $\mathrm{H}_{2}$ and $\mathrm{H}_{3}$ only have de terms, and $\mathrm{H}_{2}=\mathrm{H}_{3}^{\dagger}$. The elements in $\mathrm{H}_{2}$ and $\mathrm{H}_{3}$ contain Rabi coupling terms (half of the anticrossing energy over $\hbar$ ), and formally, these blocks are obtained by $\left\langle i\left|\mathrm{H}_{\mathrm{TB}}-\mathrm{H}_{\mathrm{EXT}}\right| j\right\rangle$, where $\mathrm{H}_{\mathrm{TB}}$ is the tight-binding Hamiltonian, and $\mathrm{H}_{\mathrm{EXT}}$ is the Hamiltonian of the extended structure. In this work, we use the approximation for Rabi coupling terms from [30] and [31], which was also used in [26].

The time evolution of the density matrix in (3) is described by the Liouville equation:

$$
\frac{d \rho}{d t}=-\frac{i}{\hbar}[\mathrm{H}, \rho]-\left(\frac{d \rho}{d t}\right)_{\text {relax }} .
$$

For intramodule transport, we can use the semiclassical scattering rates obtained using Fermi's golden rule, and for intermodule transport, we need to include scattering, which only changes the phase between two states without causing depopulation [13]. This is the main difference between the RE and DM approaches: the RE uses Fermi's golden rule for interperiod transport as well, while in the DM, we assume that resonant tunneling will occur at the Rabi frequency through the injection barrier, which is described by the Hamiltonian blocks $\mathrm{H}_{2}$ and $\mathrm{H}_{3}$, and that states additionally change their phase during the interperiod transport. The relaxation term in (4) has the symbolic form $\rho / \tau_{\text {relax }}$, but we can represent $1 / \tau_{\text {relax }}$ as

$$
\tau_{\text {relax }}^{-1}=\left(\begin{array}{ccc}
\left(1 / \tau, 1 / \tau_{\|}^{\prime \prime}\right) & 1 / \tau_{\|} & 0 \\
1 / \tau_{\|} & \left(1 / \tau, 1 / \tau_{\|}^{\prime \prime}\right) & 1 / \tau_{\|} \\
0 & 1 / \tau_{\|} & \left(1 / \tau, 1 / \tau_{\|}^{\prime \prime}\right)
\end{array}\right)
$$

where the notation $\left(1 / \tau, 1 / \tau_{\mu}^{\prime \prime}\right)$ means that diagonal blocks contain both the intraperiod lifetimes $(\tau)$ and the dephasing times $\left(\tau_{||}^{\prime \prime}\right)$, while off-diagonal blocks contain only the dephasing times $\left(\tau_{\|}\right)$between the states of different periods. Note that, in (5), we directly implemented the translation invariance of the system. These blocks have the following form:

$$
\begin{aligned}
\left(\frac{\rho_{1}}{\tau}\right)_{i i} & =\frac{\rho_{1_{i i}}}{\tau_{i}}-\sum_{i \neq j} \frac{\rho_{1_{j j}}}{\tau_{j i}} \\
\left(\frac{\rho_{1}}{\tau}\right)_{i j} & =\frac{\rho_{1_{i j}}}{\tau_{\|_{i j}}} \\
\frac{1}{\tau_{\|_{i j}}} & =\frac{1}{2 \tau_{i}}+\frac{1}{2 \tau_{j}}+\frac{1}{\tau_{i i}}+\frac{1}{\tau_{j j}}-\frac{2}{\sqrt{\tau_{i i}^{\mathrm{IFR}} \tau_{j j}^{\mathrm{IFR}}}}
\end{aligned}
$$

where $\tau_{i}=\left(\sum 1 / \tau_{i j}\right)^{-1}$ are the state lifetimes and $\tau_{i j}$ represent total intrasubband scattering lifetimes due to LO, AP, AD, II, and IFR mechanisms, obtained by thermally averaged Fermi golden rule calculations [32]. Dephasing times $\tau_{\|_{i j}}$ do not depend on intersubband processes; thus, formulation in (6) applies in the same manner to all blocks in the system in (5), with the note that dephasing occurs between two different states; thus, the main diagonal of $1 / \tau_{\|}^{\prime \prime}$ is filled with zeros, or in other words $1 / \tau_{\|}^{\prime \prime}=1 / \tau_{\|}-\operatorname{diag}\left(1 / \tau_{\|}\right)$ 


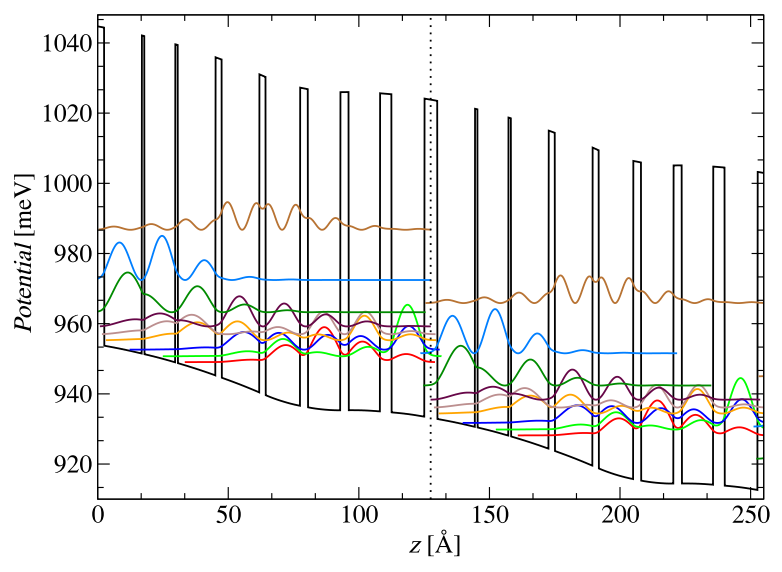

Fig. 1. Band diagram and wave function plot for the exemplar BTC QCL. The layer thicknesses, starting with the injection barrier (until dotted horizontal line), are 5.0/14.4/1.0/11.8/1.0/14.4/2.4/14.4/2.413.2/3.0/12.4/3.2/12.0/4.4/12.6 nm, $\mathrm{Al}_{0.1} \mathrm{Ga}_{0.9} \mathrm{As}$ barriers are shown in bold, and wells doped to $1.3 \times 10^{16} \mathrm{~cm}^{-3}$ are underlined.

Due to the symmetry of the system, it is possible to obtain input for (4) by considering only one period of the QCL structure in a tight-binding scheme. Eigenenergies and the scattering rates can, therefore, be calculated by a thermally balanced Schrödinger-Poisson self-consistent approach and adjacent periods can be added by translation due to the applied bias. When a three-period structure is formed, we can calculate Rabi coupling terms as in [30] and [31] (note that it applies $\mathrm{H}_{2}=\mathrm{H}_{3}^{\dagger}$ ). Our model primarily focuses on the resonant tunneling in the injection barrier, and this is motivated by the work in [13]. It is possible to generalize this approach for intraperiod resonant tunneling by sequencing the barriers of interest and creating subperiods within one period of the QCL structure, similar to [22].

Our choice of basis and our results, therefore, depend on the sequence of layers in the period, which should be considered from the injection barrier. The tight binding scheme in the DM model assumes thick padding on either side of the band diagram, in order to ensure decay of the wave functions, as can be seen in Fig. 1. RE eigenenergies and wave functions are chosen in a different manner; a two-period structure is considered in the Schrödinger-Poisson self-consistent approach, which yields both intraperiod and interperiod rates. Corresponding energies and wave functions are chosen by analyzing their localization within the period. For this reason, RE and DM wave functions can differ, and comparison of the methods can be done only in terms of the final results.

For the DM model, each block in (2), (3), and (5) is an $N \times N$ matrix, and substitution into (4) is not straightforward. The issue with the Hamiltonian in (2) is that the UU period has no interaction with the period above it, and analogously, the DD does not interact with the period below it, or in other words, the boundary conditions of the problem are not properly set. This is explained more extensively in the Appendix.

The solution of this problem lies in consideration of a general structure with $Q$ periods. If we construct the Hamiltonian in (2) with $Q$ periods, the Hamiltonian and the corresponding density matrix in (3) will become tridiagonal $Q \times Q$ matrices, and the commutator term in (4) will become a pentadiagonal matrix (shown in the Appendix). Equations on the main diagonal of this matrix would only differ at the endpoints, and this means that we neglected the boundaries (contacts) of the QCL structure and assumed instantaneous injection of the current into the device. In this work, we will use this approximation and assume that $Q \rightarrow$ $\infty$, which will be useful during the derivation of output (current density and gain profile) from this model. The infinite period approach was also used in [27] and [28], and here, we include full discussion of its implementation for any QCL structure. It is interesting to note that a $Q \times Q$ QCL model when $Q$ is finite can also be developed, but this increases modeling complexity and brings the need for a more extensive model. In reality, QCL periods do not experience same bias conditions, and domain formation effects can even cause some of the periods not to be active. Therefore, the model presented in this work represents an average effect, and in the next section, we shall show that it matches experimental results remarkably well.

The infinite approach in (4) yields the following system of equations:

$$
\begin{aligned}
\frac{d \rho_{1}}{d t} & =-\frac{i}{\hbar}\left(\left[\mathrm{H}_{1}, \rho_{1}\right]+\left[\mathrm{H}_{3}, \rho_{2}\right]+\left[\mathrm{H}_{2}, \rho_{3}\right]\right)-\frac{\rho_{1}}{\tau}-\frac{\rho_{1}}{\tau_{\|}} \\
\frac{d \rho_{2}}{d t} & =-\frac{i}{\hbar}\left(\left[\mathrm{H}_{2}, \rho_{1}\right]+\left[\mathrm{H}_{1}, \rho_{2}\right]+e K L_{\mathrm{P}} \rho_{2}\right)-\frac{\rho_{2}}{\tau_{\|}} \\
\frac{d \rho_{3}}{d t} & =-\frac{i}{\hbar}\left(\left[\mathrm{H}_{3}, \rho_{1}\right]+\left[\mathrm{H}_{1}, \rho_{3}\right]-e K L_{\mathrm{P}} \rho_{3}\right)-\frac{\rho_{3}}{\tau_{\|}} .
\end{aligned}
$$

Current density is extracted as the expectation value of the average drift velocity [33]:

$$
\mathrm{J}=\frac{\mathrm{i} e n_{2 \mathrm{D}}}{\hbar L_{\mathrm{P}}}\left[\mathrm{H}, \mathbf{Z}_{\mathrm{S}}\right]
$$

where $n_{2 \mathrm{D}}$ is the sheet population density and $Z_{\mathrm{S}}$ is the dipole matrix (shown in the Appendix) that corresponds to the Hamiltonian matrix $\mathrm{H}$. Due to the symmetry of the $\mathrm{QCL}, \mathrm{Z}_{\mathrm{S}}$ is a block-diagonal matrix, whose diagonal contains equal blocks $Z$ that correspond to the central period and offset $\pm k L_{\mathrm{P}}$ for adjacent periods due to their spatial shift from CC, where if we chose odd number of periods (the model does not depend on this, but this choice is convenient) $k=0 \ldots(Q-1) / 2$.

The expectation value of $\mathbf{J}$ is calculated as $\operatorname{Tr}(\rho \mathbf{J})$, and since we are considering an infinite structure, the current density is

$$
\begin{aligned}
& j= \lim _{Q \rightarrow \infty} \frac{\operatorname{Tr}(\rho \mathbf{J})}{Q} \\
&=\frac{\mathrm{i} e n_{2 \mathrm{D}}}{\hbar L_{\mathrm{P}}} \operatorname{Tr}\left(\rho_{1}\left[\mathrm{H}_{1}, \mathbf{Z}\right]+\rho_{2}\left[\mathrm{H}_{3}, \mathbf{Z}\right]\right. \\
& \\
&\left.\qquad \quad+\rho_{3}\left[\mathrm{H}_{2}, \mathbf{Z}\right]+L_{\mathrm{P}}\left(\mathrm{H}_{2} \rho_{3}-\rho_{2} \mathrm{H}_{3}\right)\right) .
\end{aligned}
$$

The trace in (9) passes through every term separately, and it also has a cyclic property (when trace of product of matrices is needed), which can be applied to commutator terms as $\operatorname{Tr}\left(\rho_{i}\left[\mathbf{H}_{i}, \mathbf{Z}\right]\right)=-\operatorname{Tr}\left(\mathbf{Z}\left[\mathbf{H}_{i}, \rho_{i}\right]\right)$. It can be seen that the first three terms in (9) directly correspond to the terms in the first 
equation in (7); therefore, we can also use the following expression for current density calculation:

$$
\begin{aligned}
j= & \frac{e n_{2 \mathrm{D}}}{L_{\mathrm{P}}} \operatorname{Tr}\left(\mathbf{Z}\left(\frac{d \rho_{1}}{d t}+\frac{\rho_{1}}{\tau}+\frac{\rho_{1}}{\tau_{\|}}\right)\right) \\
& +\frac{\mathrm{i} e n_{2 \mathrm{D}}}{\hbar} \operatorname{Tr}\left(\mathrm{H}_{2} \rho_{3}-\rho_{2} \mathrm{H}_{3}\right) .
\end{aligned}
$$

Gain can be extracted from $j$ by considering harmonic response of the DM terms to a light field $A_{\text {inc }}=A_{0} \mathrm{e}^{\mathrm{i} \omega t}$, and this is calculated as $-\Im(\varepsilon) \omega / n_{\mathrm{r}} / c$, where $n_{\mathrm{r}}$ is the refractive index of the material, and $\varepsilon$ is the complex permittivity of electron gas, which is calculated as $\varepsilon=j_{\text {ac }} / \frac{d E}{d t}$. Gain can also be calculated from the optical susceptibility, which equals $\chi_{\mathrm{opt}}=\frac{e n_{2 \mathrm{D}}}{\varepsilon_{0} A_{0} L_{\mathrm{P}}} \operatorname{Tr}\left(\rho_{1}^{\text {ac }} \mathbf{Z}\right)$ and gain is $g=-\Im\left(\sqrt{\varepsilon_{s}+\chi_{o p t}} \frac{w}{c}\right) \approx$ $-\Im\left(\chi_{\text {opt }} \frac{\omega h_{r}}{c}\right.$. Both of these approaches are identical; the first one was used in [26] and the second in [27]. In this work, we will use the latter; thus, the gain is calculated as

$$
g=-\frac{w e n_{2 D}}{\varepsilon_{0} A_{0} n_{r} c L_{\mathrm{P}} \hbar} \operatorname{Tr}\left(\rho_{1}^{A C} Z\right) .
$$

It is important to note that the unknowns in (7) are matrices, and due to the commutator terms, solving this system is not straightforward. This system becomes more demanding when the NRWA approach is used; every unknown in the system is assumed to have three terms ( $\mathrm{DC}, \mathrm{AC}+$, and $\mathrm{AC}-$ ), and the system in (7) expands to $9 \times 9$ system of equations with $9 N^{2} \times 9 N^{2}$ unknowns. The computational complexity of the model significantly rises with the number of states and also influences the accuracy of the solution.

A common approach for systems like that in (7) is to introduce superoperator formalism [13], [27], [28]. This turns the system in (7) into a linear system. It is important to note that such an approach should not be directly applied to (4) due to the issue with boundary conditions of the model we discussed earlier. In this work, we will not treat (7) with this formalism but rather explain the solving process from mathematical standpoint.

Systems that take the form $A X B$ in which the unknown $X$ is a matrix of size $N \times N$ can be turned into linear system as $A \otimes B^{T} X^{\prime}$, where $X^{\prime}$ is a column vector whose elements are taken row by row from matrix $X$ and operation $\otimes$ is the Kronecker tensor product. In our case, we have commutators, which linearize as $\left[H_{i}, \rho_{i}\right] \rightarrow L_{i}=\left(H_{i} \otimes I-I \otimes H_{i}^{T}\right) \rho_{i}^{\prime}$. Every commutator in (7) follows this formulation, while the linearization of the relaxation terms is different. The tensor of the form $\frac{\rho_{i j}}{\tau_{\|_{i j}}}$ linearizes to a diagonal $N^{2} \times N^{2}$ matrix by unpacking elements of $\frac{1}{\tau_{\|}}$row by row and placing them on the main diagonal of the $N^{2} \times N^{2}$ matrix. The tensor form of $\frac{\rho_{1}}{\tau}$ linearizes as illustrated in [27]. Visually, this can be described by writing down transpose of $\frac{1}{\tau}$ as a sum of $N^{2} \times N^{2}$ matrices. The first matrix of this sum would be $N \times N$ matrix, which only has element $i$ in the $i$ th position, etc. The next step is packing those matrices (terms of the aforementioned sum) in the corresponding positions in the $N^{2} \times N^{2}$ matrix. Even though this expansion is cumbersome, its numerical implementation is simple.

The output of interest is dc current, and in this case, the derivative in (10) is zero. When (6) is substituted into (10), we obtain

$$
\begin{aligned}
j^{\mathrm{DC}}= & \frac{e}{L_{\mathrm{P}}}\left(\sum_{i} \sum_{j \neq i} n_{i}\left(\frac{Z_{i i}-Z_{j j}}{\tau_{i j}}\right)\right) \\
& +\frac{e n_{2 D}}{L_{\mathrm{P}}} \operatorname{Tr}\left(\frac{Z \rho_{1}^{\mathrm{DC}}}{\tau_{\|}}+L_{\mathrm{P}}\left(\mathrm{H}_{2} \rho_{3}-\rho_{2} \mathrm{H}_{3}\right)\right) .
\end{aligned}
$$

In (12), we made use of the expression for the state lifetime as $\frac{1}{\tau_{i}}=\sum \frac{1}{\tau_{i j}}$ and the fact that population of state $i$ is given by $\rho_{i i}^{\mathrm{DC}} n_{2 D}$. The first term in (12) represents an expression for calculation of current density in the RE approach. The only difference is that in (12), $\tau_{i j}$ represents intramodule scattering rates only, while in RE models, intermodule rates would also be taken into account with the same expression. This means that if RE current was written as $j^{R E}=j_{\text {intra }}+j_{\text {inter }}$, the DM current would be $j^{D M}=j_{\text {intra* }}+j_{\text {interDM }}$, where $j_{\text {interDM }}$ corresponds to the second term in (12). Note that $j_{\text {intra* }}$ is not exactly equal to $j_{\text {intra }}$ due to the fact that wave functions for the RE and the DM models are obtained differently, but this expression still illustrates the main difference (and underlying physics) between these approaches.

The DM model can obtain $L-I$ characteristics by iteratively changing the optical field amplitude $A_{0}$ in $\mathrm{H}^{\text {ac }}$ once the material gain becomes equal to the loss. Increase of $A_{0}$ causes any excess gain to drop to the threshold value. Optical intensity is calculated as $c \epsilon_{0} n_{r} A_{0}^{2} / 2$, and this is translated by the corresponding device dimensions to the optical power.

The NRWA approach allows us to avoid direct time-domain solving of (7), and it represents the main reason why a priori knowledge of the upper and the lower lasing state is not needed, contrary to other DM models [12], [19], [22] that either use perturbation theory or add a coherent correction to the RE approach output and model the gain of the device as a Lorentzian function. Limitations of this model may become apparent with structures where intraperiod resonant tunneling cannot be neglected or structures where the nearest neighbor approximation for the period transport is invalid. It is also important to note that the system in (7) can be combined with Maxwell equations (similar to [24]) for the applications of interest, and that temperature effects of lattice self-heating could also be included, which we will discuss in the following section.

\section{NUMERICAL RESULTS}

To demonstrate the validity of the DM and the RE models, we apply them to a structure similar to the one in [34], shown in Fig. 1. The band diagram design in Fig. 1 predicts emission at $2.06 \mathrm{THz}$ at $20 \mathrm{~K}$. The $13.6 \mu \mathrm{m}$ active region is sandwiched between an upper 90-nm thick $\mathrm{n}+\mathrm{GaAs}$ layer (doping level was $n \approx 5 \times 10^{18} \mathrm{~cm}^{-3}$ ), and a lower 600-nm thick $\mathrm{n}+\mathrm{GaAs}$ layer $\left(n \approx 1.3 \times 10^{16} \mathrm{~cm}^{-3}\right)$. The latter layer allows the wafer to be processed into single metal waveguide.

The band diagram in Fig. 1 was calculated by full self-consistent Schrödinger-Poisson energy-balanced scattering transport simulations [35], [36]. 


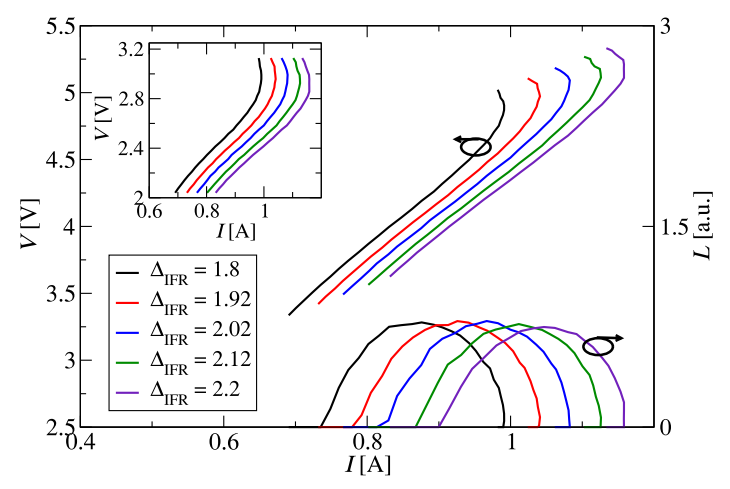

Fig. 2. DM model $V-I$ characteristics for various values of IFR height $\Delta_{\mathrm{IFR}}$; IFR correlation length was set to $\Lambda=80 \AA$. The contact resistance of $2.15 \Omega$ is also included. Inset shows the $V-I$ results without the contact resistance. $L-I$ results are also presented for every $\Delta_{\mathrm{IFR}}$ accordingly and normalized to the unit value.

In our modeling procedure, we use three fitting parameters in order to compare our results with the experimental data: 1) total loss in the laser cavity; 2) IFR parameters; 3) contact resistance.

1) Cavity loss directly determines the lasing threshold, and this can be estimated by various waveguide models. In this work, we use a 1-D transfer matrix calculation of waveguide modes, with a Drude-Lorentz model for permittivities, as described in [37] and [38].

2) The IFR parameters are not experimentally known and can vary from structure to structure. This brings the need for additional sweeping of these parameters in the simulation, and the optimal values depend on the fitting goals: obtaining threshold fit, peak power fit, or power roll over fit (ideally the entire $V-I$ and $L-I$ fit).

3) Contact resistance parameter can significantly shift $V-I$ characteristics, and approximate values can be obtained experimentally. Typical values are several ohms [39].

For the device in Fig. 1, we used models from [37] and [38] to estimate the loss to $32.42 \mathrm{~cm}^{-1}$ in pulsed operation at a cold finger temperature of $20 \mathrm{~K}$. This design has an experimental dynamic range of $0.837-1.08 \mathrm{~A}$, and IFR parameters are found to match threshold current assuming the calculated cavity loss value; this is illustrated in Fig. 2. Then, we search for the value of contact resistance that fits the experimental data; this is estimated to $2.15 \Omega$.

The $V-I$ and $L-I$ characteristics calculated with the DM model for different IFR roughness heights are shown in Fig. 2. It should be noted that the width of the $L-I$ curve remains relatively unchanged with the increase of IFR scattering. Current is noted to be more sensitive to changes in IFR scattering. The width can be changed by altering the loss value. Our model does not include the self-heating of the lattice above cold finger temperature, and therefore, only pulsed operation can be properly modeled, while $\mathrm{CW}$ operation would require an appropriate thermal model [40].

The RE fitting procedure requires a different fitting approach from the DM due to the differences in the interperiod transport. Our RE model does not include a photon interaction term, and producing $L-I$ results is not straightforward. Therefore, in this work, we focus on the DM model and its full comparison with the experimental results. Note that the IFR parameters used

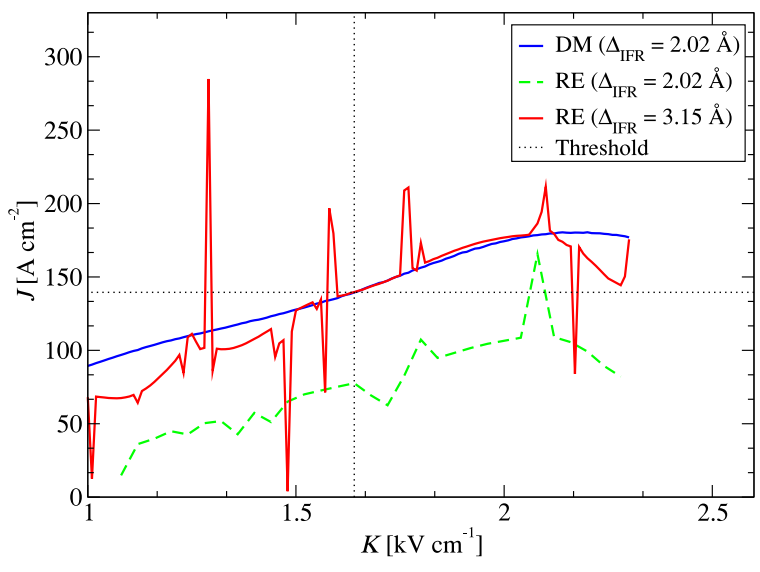

Fig. 3. Current density versus electric field calculated using RE (red) and DM models (blue). Results were fitted to threshold point at $\left(K_{t}, J_{t}\right)=$ $\left(1.64 \mathrm{kV} / \mathrm{cm}, 139.5 \mathrm{~A} / \mathrm{cm}^{2}\right)$. The RE model requires $\Delta_{\mathrm{IF} \mathrm{R}}^{\prime}=3.15 \AA$ for threshold fit, while the DM needs $\Delta_{\mathrm{IFR}}=2.02 \AA$. Results for $\Delta_{\mathrm{IFR}}^{\prime}=2.02 \AA$ for the $\mathrm{RE}$ are also displayed (dashed green).

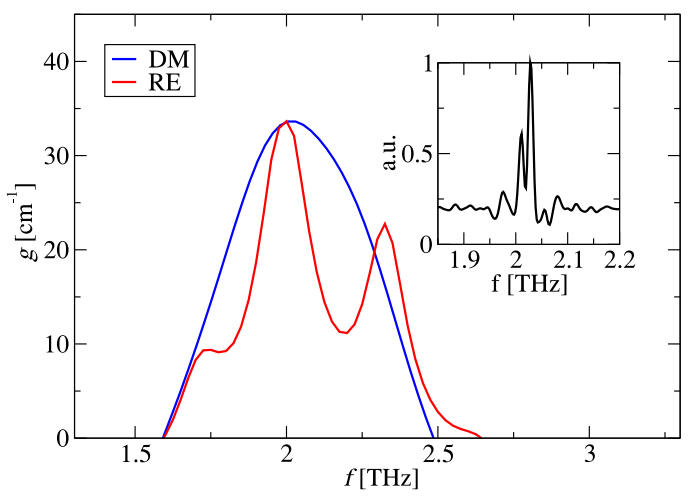

Fig. 4. Gain versus frequency from RE (red) and DM models (blue). Results were fitted to loss of $32.42 \mathrm{~cm}^{-1}$ at threshold point $\left(K_{t}, J_{t}\right)=$ $\left(1.64 \mathrm{kV} / \mathrm{cm}, 139.5 \mathrm{~A} / \mathrm{cm}^{2}\right)$ at $20 \mathrm{~K}$. Inset shows experimental spectral measurement at $20 \mathrm{~K}$ at threshold bias.

in the DM will not be exactly the same due to the different approaches in interperiod transport and different basis wave functions.

Fig. 3 presents current density versus electric field dependence obtained by RE and DM models, while Fig. 4 shows gain dependence on frequency at lasing threshold when gain becomes equal to loss of $32.42 \mathrm{~cm}^{-1}$.

The DM results show a smooth dependence in Fig. 3, contrary to the RE model that exhibits nonphysical spikes. The structure is expected to begin to lase at $K=1.64 \mathrm{kV} / \mathrm{cm}$ in both methods. The results in Fig. 3 were fitted differently only in terms of $\Delta_{\text {IFR }}$. We also presented the $K-J$ dependence when all fitting parameters are equal (green-dashed line in Fig. 3); however, this result does not reach the threshold current density and overestimates the gain value as well. For the RE approach, we found that the value of $\Delta_{\mathrm{IFR}}^{\prime}=3.15 \AA$ fits the desired current density and the loss value.

Spectral results in Fig. 4 show that the DM approach exhibits broader spectral line, with one hump clearly visible. Very good agreement is observed in terms of lasing frequency of $2.06 \mathrm{THz}$.

In order to closely examine what happens around a spike occurrence, we will focus around the point $K=1.3 \mathrm{kV} / \mathrm{cm}$ 


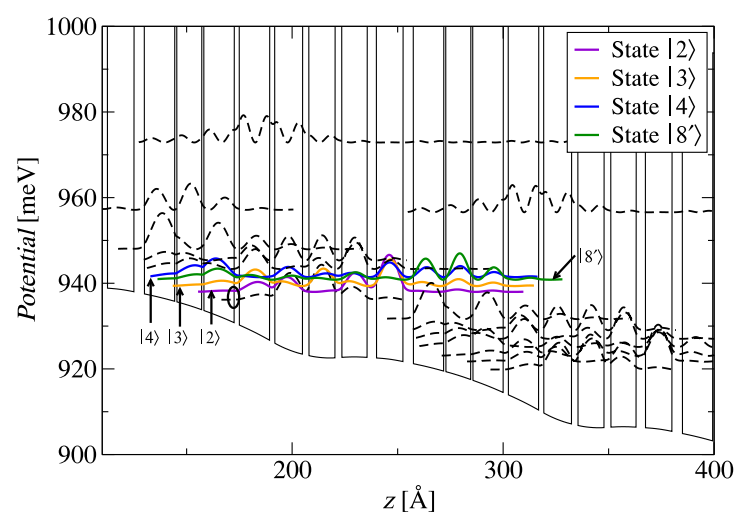

(a)

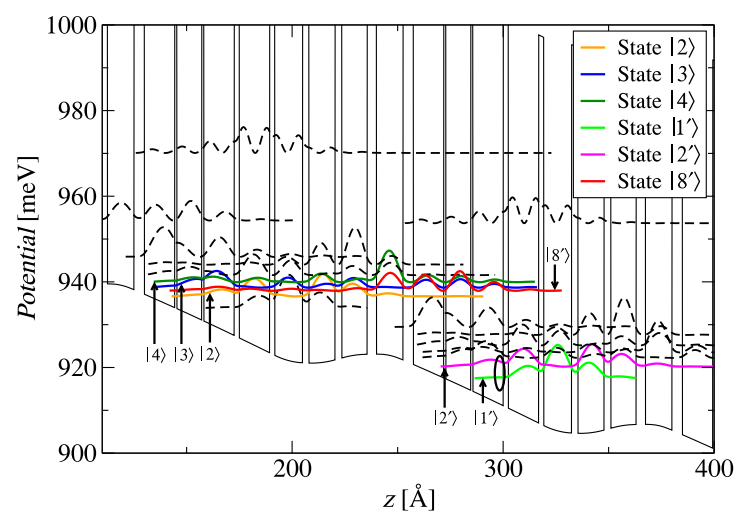

(b)

Fig. 5. Band diagram and the corresponding wave functions at (a) $K=$ $1.28 \mathrm{kV} / \mathrm{cm}$ (nonspike point) and (b) $K=1.29 \mathrm{kV} / \mathrm{cm}$ (spike point), obtained by RE model. States of interest are labeled as $|i\rangle$ if they belong in the left period and $\left|i^{\prime}\right\rangle$ if they belong in the adjacent period to the right. Circles indicate the change of the second state in the spike point.

in Fig. 3. In Fig. 5, we present a band diagram and the corresponding wave functions, with the RE model, at the standard point $K=1.28 \mathrm{kV} / \mathrm{cm}$ and at the unphysical spike point $K=1.29 \mathrm{kV} / \mathrm{cm}$. In Fig. 5(a), we can see that the fourth state from the left period $|4\rangle$ is nearly aligned with the eighth state $\left|8^{\prime}\right\rangle$ from the adjacent period at $K=1.28 \mathrm{kV} / \mathrm{cm}$. At $K=1.29 \mathrm{kV} / \mathrm{cm}$ [see Fig. 5(b)], position of the states has changed. State $\left|8^{\prime}\right\rangle$ is now nearly aligned with the state $|2\rangle$; however, compared to the situation at $K=1.28 \mathrm{kV} / \mathrm{cm}$, it seems that the state $|2\rangle$ does not exist in Fig. 5(b) (indicated by circles). Effectively, state $\left|8^{\prime}\right\rangle$ from the second period in Fig. 5(a) became state $|4\rangle$ in the first period in Fig. 5(b); this then caused the shift of states when two figures are compared $\left[\left|8^{\prime}\right\rangle_{a} \rightarrow|4\rangle_{b}\right.$, $|4\rangle_{a} \rightarrow|3\rangle_{b}$, and $|3\rangle_{a} \rightarrow|2\rangle_{b}$; changes are purposely colored the same in Fig. 5(a) and (b). Note that in Fig. 5(b), the QCL period gained an additional state $\left(\left|8^{\prime}\right\rangle\right)$. A similar situation to that in Fig. 5 occurs at every unphysical spike point observed in Fig. 3. This is consistent with the characteristic RE model feature of instantaneous transport due to extended wave functions. This depicts that the RE model predicts unphysically high currents due to instantaneous nonlocal transport for spatially separated resonances, which causes detrimental effects in the electron transport. Note that depending on the step size of the electric field in the simulation, more spikes would occur due

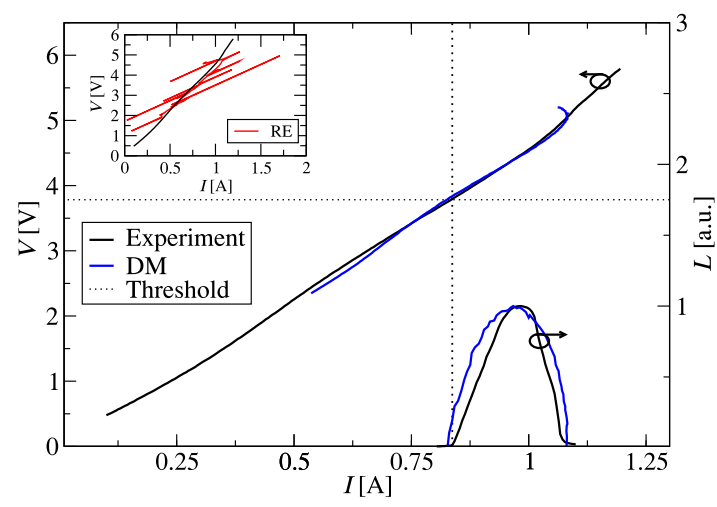

Fig. 6. $L-I-V$ characteristics of the BTC device at $20 \mathrm{~K}$ for pulsed operation. Contact resistance is $2.15 \Omega$. Both DM model fit (blue) and RE model fit (inset, red) were fitted to experimental threshold at $\left(I_{t}, V_{t}\right)=(0.83 \mathrm{~A}, 3.78 \mathrm{~V})$. Experimental $L-I$ data are presented along DM $L-I$ data and normalized in arbitrary units.

to a higher probability of producing state alignment (it is also possible to have multiple anticrossings at one point). This effect is caused by the inability of the RE model to describe the QCL structure when anticrossing occurs, and whenever the alignment of two states from adjacent periods takes place, nonphysical spike in the current density-electric field (and hence in the corresponding current-voltage) characteristic of the device is displayed.

In order to obtain comparison with the experimental voltagecurrent $(V-I)$ characteristics, the axes in Fig. 3 need to be scaled by the corresponding device dimensions. Fig. 6 shows how the $\mathrm{RE}$ and the DM models compare to the experimental output.

Based on the design in Fig. 1, L1071_C6_S3 THz QCL wafer was grown via molecular beam epitaxy (MBE) in an Oxford Instruments V-80 H MBE system on a semiinsulating GaAs substrate. The laser material consists of 110 repeat periods of $\mathrm{Al}_{0.1} \mathrm{Ga}_{0.9} \mathrm{As}$ (Al: $10 \%$ ) heterostructures forming the $\sim 13.6 \mu \mathrm{m}$-thick active region (see layer structures given in Fig. 1). The device was processed into single-metal semiinsulating surface-plasmon ridge waveguide of $200-\mu \mathrm{m}$ width and cleaved to a length of $3 \mathrm{~mm}$. Fig. 6 compares the measured and calculated optical power-current-voltage $(L-I-V)$ characteristics at $20 \mathrm{~K}$ with the DM approach. Good agreement is obtained once the (realistic) contact resistance of $2.15 \Omega$ is taken into account. Note that the calculated $L-I$ is for the optical power within the cavity, which will differ from the experimental value due to the sensitivity of detector. The shape of experimental data cannot match the theoretical one due to the measurement setup (the beam is collimated onto the detector). The presented experimental data do not show the optical power directly, but rather the voltage variations in the measuring device. For that reason, both experimental and theoretical results were normalized to 1 in Fig. 6, and emphasis of the result is put on the dynamic range.

In addition to good agreement between the measured and the calculated $V-I$ characteristics, the value of the driving current when the structure begins to roll over (around $1.08 \mathrm{~A}$ ), agrees very well with the experimental dynamic range due to the fitting procedure we presented in Fig. 2. This behavior is caused by misalignment of subband levels, i.e., reduced electron transports throughout the injection barrier. 


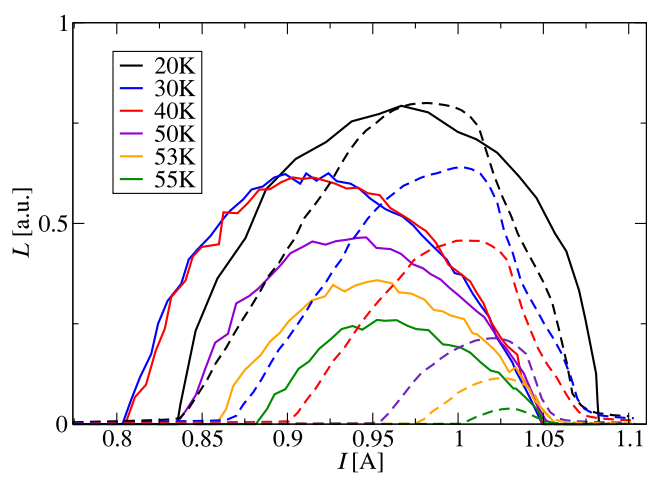

Fig. 7. $L-I$ characteristics for different cold finger temperatures. Full lines represent simulation result, while dashed lines represent the experimental measurement. Scaling was performed, so peak ratio is consistent in both results. We included the dependence of cavity loss on temperature in accordance with [37] and [38]: $g_{\mathrm{th}}=32.42 \mathrm{~cm}^{-1}(20 \mathrm{~K}), 32.13 \mathrm{~cm}^{-1}(30 \mathrm{~K}), 31.91 \mathrm{~cm}^{-1}(40 \mathrm{~K})$, $31.75 \mathrm{~cm}^{-1}(50 \mathrm{~K}), 31.71 \mathrm{~cm}^{-1}(53 \mathrm{~K}), 31.68 \mathrm{~cm}^{-1}(55 \mathrm{~K})$.

Another source of discrepancy is that self-heating is not included in our model due to its computational complexity. We expect that agreement would be improved further if included, as gain would reduce faster at higher currents. Note that this effect needs to be included for pulsed operation at higher temperatures as well. To illustrate this, in Fig. 7, we present the $L-I$ simulations for different temperatures while keeping $\Delta_{\mathrm{IFR}}=2.02 \AA$ and contact resistance of $2.15 \Omega$.

In Fig. 7, we included the dependence of the waveguide loss on the temperature discussed in [37], which slightly decreases, and that is one of the reasons why threshold currents do not fully match the experiment. We also notice that the model gives similar dynamic ranges at 20,30, and $40 \mathrm{~K}$, and that the sharp drop of peak power is only noticeable at higher temperatures. Such behavior is related to exponential drop of the upper lasing level state lifetime with temperature in BTC structures [41], which becomes significant in the similar temperature range as in Fig. 7. It can also be noted that at higher temperatures, the relative rate of theoretical power peaks match the experimental ones. The main reason for the mismatch of theoretical and experimental threshold current may also be attributed to the lack of a full thermal model within these calculations, which assume that heat sink temperature equals the lattice temperature in the device.

The self-heating is certainly larger in CW operation, and the sensitivity of the gain on temperature is larger at higher temperatures, implying that the discrepancy between theoretical and experimental results will then be larger as well. Interestingly, dynamic range at cold finger temperature of $20 \mathrm{~K}$ for $\mathrm{CW}$ operation is $0.971-1.1 \mathrm{~A}$, which matches the case of $53 \mathrm{~K}$ in Fig. 7, and this gives an idea about the self-heating.

Additionally, the fitting procedure illustrated in Fig. 2 depends on the desired modeling outcome. We noticed in Fig. 2 that for the set value of loss, the width of dynamic range roughly remains the same. This device has much narrower dynamic range for $\mathrm{CW}$ operation (0.971-1.07 A), and even though it is possible to fit the threshold value by a higher value of IFR parameters, the dynamic range would be overestimated greatly. This effect happens due to the lack of the inclusion of lattice self-heating due to the electrical power in our model. It is important to underline that IFR parameters do not depend on the temperature, and if they are swept to match $\mathrm{CW}$ threshold, that value would compensate the other scattering processes that do depend on the temperature.

\section{CONCLUSION}

We have presented a DM model for QCLs, which considers an infinite number of QCL periods, which interact within the nearest neighbor approximation. We derived model output (current density and gain calculation) and discussed the link with the RE model. The mathematical expression we provided shows a clear difference between transport calculation in RE and DM approaches.

We outlined the modeling approach and discussed the fitting parameters, which allow the comparison of the model with experimentally analyzed structures in pulsed mode operation.

When applied to BTC structures, this model provides smooth results in contrast to the RE. The results show very good agreement with experimental data in terms of current-voltage profile, lasing frequency, and current-optical power profile and show promise for application in QCL device optimization.

\section{APPENDIX}

If we substitute (2) and (3) into (4) and label the commutator term as $M$, and rearrange this equation so that $M$ is kept on the left-hand side, we obtain the following expression:

$$
\begin{aligned}
M & =\left[\begin{array}{ccc}
C_{U} & U & E_{2} \\
D & C & U \\
E_{3} & D & C_{D}
\end{array}\right] \\
& =i \hbar \frac{d}{d t}\left[\begin{array}{ccc}
\rho_{1} & \rho_{2} & 0 \\
\rho_{3} & \rho_{1} & \rho_{2} \\
0 & \rho_{3} & \rho_{1}
\end{array}\right]+i \hbar\left(\frac{d \rho}{d t}\right)_{\text {relax }}
\end{aligned}
$$

where the terms in $M$ are

$$
\begin{aligned}
C_{U} & =\left[\mathrm{H}_{1}, \rho_{1}\right]-\rho_{2} \mathrm{H}_{3}+\mathrm{H}_{2} \rho_{3} \\
C_{D} & =\left[\mathrm{H}_{1}, \rho_{1}\right]+\mathrm{H}_{3} \rho_{2}-\rho_{3} \mathrm{H}_{2} \\
C & =\left[\mathrm{H}_{1}, \rho_{1}\right]+\left[\mathrm{H}_{3}, \rho_{2}\right]+\left[\mathrm{H}_{2}, \rho_{3}\right] \\
U & =\left[\mathrm{H}_{2}, \rho_{1}\right]+\left[\mathrm{H}_{1}, \rho_{2}\right]+\operatorname{eKL} \rho_{2} \rho_{2} \\
D & =\left[\mathrm{H}_{3}, \rho_{1}\right]+\left[\mathrm{H}_{1}, \rho_{3}\right]-\operatorname{eKL}_{\mathrm{P}} \rho_{3} \\
E_{2} & =\left[\mathrm{H}_{2}, \rho_{2}\right] \\
E_{3} & =\left[\mathrm{H}_{3}, \rho_{3}\right] .
\end{aligned}
$$

Equation (A1) is Hermitian (as expected), but the equations on the main diagonal are not equal. The right-hand side of the equation has completely equal terms on the main diagonal, while the left-hand side does not; therefore, the system is mathematically overdetermined. We have three unknowns and seven equations: $\rho_{1}$ (three equations), $\rho_{2}$ (one equation), and $\rho_{3}$ (one equation) and two extra equations $\left(E_{2}\right.$ and $\left.E_{3}\right)$. These extra equations describe the coupling between UU and DD periods, which can be neglected; however, this still leaves five equations and three unknowns. 
This can be resolved by constructing a $Q \times Q$ system, and then, (A1) expands to a pentadiagonal matrix. In this case, the commutator term $M_{Q \times Q}$ has terms $C_{U}$ and $C_{D}$ at end points of the main diagonal, while all other terms on the main diagonal are equal to $C$. This occurrence is the consequence of the fact that the $Q \times Q$ Hamiltonian cannot see the period above and below it, and it needs boundary conditions. The main assumption in this work is that boundaries of the devices do not have large influence on the structure, so they can be neglected. Therefore, equations with terms $C, U$, and $D$ in (A2) need to be chosen for solving the system, and we can let $Q \rightarrow \infty$ when finding the output of the model.

The dipole matrix for $Q=3$ for our system has the form

$$
Z_{S}=\left[\begin{array}{ccc}
Z-L_{\mathrm{P}} I & 0 & 0 \\
0 & Z & 0 \\
0 & 0 & Z+L_{\mathrm{P}} I
\end{array}\right]
$$

where $I$ is an $N \times N$ identity matrix, $Z_{S}$ expands correspondingly for larger number of periods, and output of the system needs to be derived as the limit value, when $Q \rightarrow \infty$ as de- scribed in Section II. Note that $Z_{S}$ does not have any interperiod components, and this is due to the tight binding choice of the basis for our model, which was discussed in Section II.

The system is described by equations containing terms $C, U$, and $D$ in (A1); each commutator in the system linearizes as $L_{i}=$ $\left(H_{i} \otimes I-I \otimes H_{i}^{T}\right)$, and when NRWA formalism is introduced, the full system has the form shown in (A4) at the bottom of this page, where $V_{\delta}=e K L_{\mathrm{P}} I_{N^{2} \times N^{2}}$, the right-hand side consists of envelopes $\rho_{i}^{\text {ac/dc' }}$, packed rowwise in $N^{2} \times N^{2}$ column vectors, and $\tau_{\text {lin }}^{-1}$ and $\tau_{\operatorname{lin} \|}$ are linearized blocks of size $N^{2} \times N^{2}$, which in case for $N=2$ have the form

$$
\begin{aligned}
\tau_{\|}^{-1}= & {\left[\begin{array}{cc}
\tau_{\|_{11}}^{-1} & \tau_{\|_{12}}^{-1} \\
\tau_{\|_{21}}^{-1} & \tau_{\|_{22}}^{-1}
\end{array}\right] \rightarrow } \\
\tau_{\operatorname{lin} \|}^{-1}= & {\left[\begin{array}{cccc}
\tau_{\|_{11}}^{-1} & 0 & 0 & 0 \\
0 & \tau_{\|_{12}}^{-1} & 0 & 0 \\
0 & 0 & \tau_{\|_{21}}^{-1} & 0 \\
0 & 0 & 0 & \tau_{\|_{22}}^{-1}
\end{array}\right] }
\end{aligned}
$$

$$
\begin{aligned}
& \left.\left(\begin{array}{ccccccccc}
L_{1}^{\mathrm{dc}} & L_{1}^{\mathrm{ac}} & L_{1}^{\mathrm{ac}} & L_{3} & 0 & 0 & L_{2} & 0 & 0 \\
L_{1}^{\mathrm{ac}} & L_{1}^{\mathrm{dc}} & 0 & 0 & L_{3} & 0 & 0 & L_{2} & 0 \\
L_{1}^{\mathrm{ac}} & 0 & L_{1}^{\mathrm{dc}} & 0 & 0 & L_{3} & 0 & 0 & L_{2} \\
L_{2} & 0 & 0 & L_{1}^{\mathrm{dc}}+V_{\delta} & L_{1}^{\mathrm{ac}} & L_{1}^{\mathrm{ac}} & 0 & 0 & 0 \\
0 & L_{2} & 0 & L_{1}^{\mathrm{ac}} & L_{1}^{\mathrm{dc}}+V_{\delta} & 0 & 0 & 0 & 0 \\
0 & 0 & L_{2} & L_{1}^{\mathrm{ac}} & 0 & L_{1}^{\mathrm{dc}}+V_{\delta} & 0 & 0 & 0 \\
L_{3} & 0 & 0 & 0 & 0 & 0 & L_{1}^{\mathrm{dc}}-V_{\delta} & L_{1}^{\mathrm{ac}} & \\
0 & L_{3} & 0 & 0 & 0 & 0 & L_{1}^{\mathrm{ac}} & L_{1}^{\mathrm{dc}}-V_{\delta} & \\
0 & 0 & L_{3} & 0 & 0 & 0 & L_{1}^{\mathrm{ac}} & 0 & L_{1}^{\mathrm{dc}}-V_{\delta}
\end{array}\right]\right) \\
& -\left(\left[\begin{array}{ccccccccc}
\tau_{\operatorname{lin}}^{-1}+\tau_{\operatorname{lin} \|}^{-1} & 0 & 0 & 0 & 0 & 0 & 0 & 0 & 0 \\
0 & \tau_{\operatorname{lin}}^{-1}+\tau_{\operatorname{lin} \|}^{-1} & 0 & 0 & 0 & 0 & 0 & 0 & 0 \\
0 & 0 & \tau_{\operatorname{lin}}^{-1}+\tau_{\operatorname{lin} \|}^{-1} & 0 & 0 & 0 & 0 & 0 & 0 \\
0 & 0 & 0 & \tau_{\operatorname{lin} \|}^{-1} & 0 & 0 & 0 & 0 & 0 \\
0 & 0 & 0 & 0 & \tau_{\operatorname{lin} \|}^{-1} & 0 & 0 & 0 & 0 \\
0 & 0 & 0 & 0 & 0 & \tau_{\operatorname{lin} \|}^{-1} & 0 & 0 & 0 \\
0 & 0 & 0 & 0 & 0 & 0 & \tau_{\operatorname{lin} \|}^{-1} & 0 & 0 \\
0 & 0 & 0 & 0 & 0 & 0 & 0 & \tau_{\operatorname{lin} \|}^{-1} & 0 \\
0 & 0 & 0 & 0 & 0 & 0 & 0 & 0 & \tau_{\operatorname{lin} \|}^{-1}
\end{array}\right]\right)\left[\begin{array}{c}
\rho_{1}^{\mathrm{dc}^{\prime}} \\
\rho_{1}^{\mathrm{ac}+^{\prime}} e^{i \omega t} \\
\rho_{1}^{\mathrm{ac}-^{\prime}} e^{-i \omega t} \\
\rho_{2}^{\mathrm{dc}^{\prime}} \\
\rho_{2}^{\mathrm{ac}+^{\prime}} e^{i \omega t} \\
\rho_{2}^{\mathrm{ac}-^{\prime}} e^{-i \omega t} \\
\rho_{3}^{\mathrm{dc}^{\prime}} \\
\rho_{3}^{\mathrm{ac}+^{\prime}} e^{i \omega t} \\
\rho_{3}^{\mathrm{ac}-^{\prime}} e^{-i \omega t}
\end{array}\right]=\frac{d}{d t}\left[\begin{array}{c}
\rho_{1}^{\mathrm{dc}^{\prime}} \\
\rho_{1}^{\mathrm{ac}+^{\prime}} e^{i \omega t} \\
\rho_{1}^{\mathrm{ac}-^{\prime}} e^{-i \omega t} \\
\rho_{2}^{\mathrm{dd} c^{\prime}} \\
\rho_{2}^{\mathrm{ac}+^{\prime}} e^{i \omega t} \\
\rho_{2}^{\mathrm{ac}-^{\prime}} e^{-i \omega t} \\
\rho_{3}^{\mathrm{dc} c^{\prime}} \\
\rho_{3}^{\mathrm{ac}+^{\prime}} e^{i \omega t} \\
\rho_{3}^{\mathrm{ac}-^{\prime}} e^{-i \omega t}
\end{array}\right]
\end{aligned}
$$




$$
\begin{aligned}
\tau^{-1}= & {\left[\begin{array}{cc}
\tau_{11}^{-1} & \tau_{12}^{-1} \\
\tau_{21}^{-1} & \tau_{22}^{-1}
\end{array}\right] \rightarrow } \\
\tau_{\text {lin }}^{-1}= & {\left[\begin{array}{cccc}
\tau_{11}^{-1} & 0 & 0 & \tau_{12}^{-1} \\
0 & 0 & 0 & 0 \\
0 & 0 & 0 & 0 \\
\tau_{21}^{-1} & 0 & 0 & \tau_{22}^{-1}
\end{array}\right] . }
\end{aligned}
$$

Operation in (A6) visually seems as stretching of a matrix, and it can also be constructed as

$$
\begin{aligned}
\tau^{-1} & =\left[\begin{array}{cc}
\tau_{11}^{-1} & 0 \\
0 & 0
\end{array}\right]+\left[\begin{array}{cc}
0 & \tau_{12}^{-1} \\
0 & 0
\end{array}\right]+\left[\begin{array}{cc}
0 & 0 \\
\tau_{21}^{-1} & 0
\end{array}\right]+\left[\begin{array}{cc}
0 & 0 \\
0 & \tau_{22}^{-1}
\end{array}\right] \\
& =T_{11}+T_{12}+T_{21}+T_{22}, \\
\tau_{\text {lin }}^{-1} & =\left[\begin{array}{ll}
T_{11} & T_{12} \\
T_{21} & T_{22}
\end{array}\right] .
\end{aligned}
$$

Operation in (A7) generalizes analogously, the original $\tau^{-1}$ is written as a sum of the corresponding $T_{i j}$ matrices of size $N \times N$ with respect to the element position, and the linearized form is constructed by rowwise packing of those block matrices. Numerically, this operation is simply performed as $\tau_{\operatorname{lin}_{i N+i, j N+j}}^{-1}=\frac{1}{\tau_{j i}}$ and $i, j=0,1 \ldots N-1$ (for implementation in $\mathrm{C} / \mathrm{C}++$ ).

The steady-state solution can be derived from (A4) by assuming that envelopes $\rho_{i}^{\mathrm{dc} / \mathrm{ac}}$ are not time dependent. In this case, the system is linear $9 N^{2} \times 9 N^{2}$ and homogeneous. This can be simplified by turning the system into a nonhomogeneous system by using the trace condition $\sum_{i} \rho_{i i}^{\mathrm{dc}}=1$ and substituting this equation instead of any equation of the system into (A4) that targets one of the $\rho_{i i}^{\mathrm{dc}}$ elements. Note that choosing some other equation to be substituted would result in unphysical result, due to the fact that system is not diagonally dominant.

\section{ACKNOWLEDGMENT}

The numerical part of this work was undertaken on ARC2, part of the High Performance Computing facilities at The University of Leeds, Leeds, U.K.

\section{REFERENCES}

[1] R. Köhler et al., "Terahertz semiconductor-heterostructure laser," $\mathrm{Na}$ ture, vol. 417, no. 6885, pp. 156-159, 2002. [Online]. Available: http://www.nature.com/nature/journal/v417/n6885/abs/417156a.html

[2] C. Gmachl, F. Capasso, D. L. Sivco, and A. Y. Cho, " Recent progress in quantum cascade lasers and applications," Rep. Prog. Phys., vol. 64, no. 11, p. 1533, 2001. [Online]. Available: http://stacks.iop.org/0034$4885 / 64 / \mathrm{i}=11 / \mathrm{a}=204$

[3] J. Faist, F. Capasso, D. L. Sivco, C. Sirtori, A. L. Hutchinson, and A. Y. Cho, "Quantum cascade laser," Science, vol. 264, no. 5158, pp. 553-556, 1994. [Online]. Available: http://science.sciencemag.org/ content/264/5158/553

[4] A. A. Kosterev and F. K. Tittel, "Chemical sensors based on quantum cascade lasers," IEEE J. Quantum Electron., vol. 38, no. 6, pp. 582-591, Jun. 2002.

[5] G. Wysocki et al., "Widely tunable mode-hop free external cavity quantum cascade lasers for high resolution spectroscopy and chemical sensing," Appl. Phys. B, Lasers Opt., vol. 92, no. 3, pp. 305-311, 2008. [Online] Available: http://link.springer.com/article/10.1007/s00340-008-3047-x
[6] J. B. McManus, J. H. Shorter, D. D. Nelson, M. S. Zahniser, D. E. Glenn, and R. M. McGovern, "Pulsed quantum cascade laser instrument with compact design for rapid, high sensitivity measurements of trace gases in air," Appl. Phys. B, Lasers Opt., vol. 92, no. 3, p. 387, 2008. [Online]. Available: http://link.springer.com/article/10.1007/s00340-008-3129-9

[7] A. D. Rakić et al., "Swept-frequency feedback interferometry using terahertz frequency QCLs: A method for imaging and materials analysis," Opt. Exp., vol. 21, no. 19, pp. 22194-22205, 2013. [Online]. Available: http://www.osapublishing.org/abstract.cfm?uri=oe-21-19-22194

[8] M. Wienold et al., "Frequency dependence of the maximum operating temperature for quantum-cascade lasers up to $5.4 \mathrm{THz}$," Appl. Phys. Lett., vol. 107, no. 20, 2015, Art. no. 202101. [Online]. Available: http://aip.scitation.org/doi/abs/10.1063/1.4935942

[9] C. W. I. Chan, Q. Hu, and J. L. Reno, "Ground state terahertz quantum cascade lasers," Appl. Phys. Lett., vol. 101, no. 15, 2012, Art. no. 151108.

[10] G. Scalari et al., "THz and sub-THz quantum cascade lasers," Laser Photon. Rev., vol. 3, nos. 1/2, pp. 45-66, 2009. [Online]. Available: http://onlinelibrary.wiley.com/doi/10.1002/lpor.200810030/abstract

[11] S. Fathololoumi et al., "Terahertz quantum cascade lasers operating up to $\sim 200 \mathrm{~K}$ with optimized oscillator strength and improved injection tunneling," Opt. Exp., vol. 20, no. 4, pp. 3866-3876, 2012. [Online]. Available: http://www.osapublishing.org/abstract.cfm?uri=oe-20-4-3866

[12] C. Jirauschek and T. Kubis, "Modeling techniques for quantum cascade lasers," Appl. Phys. Rev., vol. 1, no. 1, 2014, Art. no. 011307. [Online]. Available: http://scitation.aip.org/content/aip/journal/apr2/1/1/ $10.1063 / 1.4863665$

[13] H. Callebaut and Q. Hu, "Importance of coherence for electron transport in terahertz quantum cascade lasers," J. Appl. Phys., vol. 98 no. 10, 2005, Art. no. 104505. [Online]. Available: http://scitation.aip.org/ content/aip/journal/jap/98/10/10.1063/1.2136420

[14] T. Schmielau and M. F. Pereira, "Impact of momentum dependent matrix elements on scattering effects in quantum cascade lasers," Phys. Status Solidi (b), vol. 246, no. 2, pp. 329-331, 2009. [Online]. Available: http://onlinelibrary.wiley.com/doi/10.1002/pssb.200880328/abstract

[15] H. Yasuda, T. Kubis, P. Vogl, N. Sekine, I. Hosako, and K. Hirakawa, "Nonequilibrium Green's function calculation for four-level scheme terahertz quantum cascade lasers," Appl. Phys. Lett., vol. 94, no. 15, 2009, Art. no. 151109. [Online]. Available: http://aip.scitation.org/doi/ full/10.1063/1.3119312

[16] A. Kolek, G. Haldaś, and M. Bugajski, "Nonthermal carrier distributions in the subbands of 2-phonon resonance mid-infrared quantum cascade laser," Appl. Phys. Lett., vol. 101, no. 6, 2012, Art. no. 061110.

[17] A. Wacker, M. Lindskog, and D. O. Winge, "Nonequilibrium Green's function model for simulation of quantum cascade laser devices under operating conditions," IEEE J. Sel. Topics Quantum Electron., vol. 19, no. 5, Sep./Oct. 2013, Art. no. 1200611.

[18] N. Vukmirović, Z. Ikonić, D. Indjin, and P. Harrison, "Quantum transport in semiconductor quantum dot superlattices: Electron-phonon resonances and polaron effects," Phys. Rev. B, Condens. Matter, vol. 76, no. 24, 2007, Art. no. 245313.

[19] R. W. Boyd, Nonlinear Optics. San Francisco, CA, USA: Academic, 2008.

[20] S. Kumar and Q. Hu, "Coherence of resonant-tunneling transport in terahertz quantum-cascade lasers," Phys. Rev. B, Condens. Matter, vol. 80, no. 24, 2009, Art. no. 245316. [Online]. Available: http://link.aps.org/ doi/10.1103/PhysRevB.80.245316

[21] E. Dupont, S. Fathololoumi, and H. C. Liu, "Simplified density-matrix model applied to three-well terahertz quantum cascade lasers," Phys. Rev. B, Condens. Matter, vol. 81, no. 20, 2010, Art. no. 205311. [Online]. Available: http://link.aps.org/doi/10.1103/PhysRevB.81.205311

[22] R. Terazzi and J. Faist, "A density matrix model of transport and radiation in quantum cascade lasers," New J. Phys., vol. 12, no. 3 , 2010, Art. no. 033045. [Online]. Available: http://stacks.iop.org/1367$2630 / 12 / \mathrm{i}=3 / \mathrm{a}=033045$

[23] G. Beji, Z. Ikonić, C. A. Evans, D. Indjin, and P. Harrison, "Coherent transport description of the dual-wavelength ambipolar terahertz quantum cascade laser," J. Appl. Phys., vol. 109, no. 1, 2011, Art. no. 013111. [Online]. Available: http://scitation.aip.org/content/aip/ journal/jap/109/1/10.1063/1.3530628

[24] P. Tzenov, D. Burghoff, Q. Hu, and C. Jirauschek, "Time domain modeling of terahertz quantum cascade lasers for frequency comb generation," Opt. Exp., vol. 24, no. 20, pp. 23232-23247, 2016. [Online]. Available: http://www.osapublishing.org/abstract.cfm?uri=oe-24-20-23232

[25] S. Barbieri, J. Alton, H. E. Beere, J. Fowler, E. H. Linfield, and D. A. Ritchie, "2.9 THz quantum cascade lasers operating up to $70 \mathrm{k}$ in continuous wave," Appl. Phys. Lett., vol. 85, no. 10, pp. 1674-1676, 2004. 
[26] T. V. Dinh, A. Valavanis, L. J. M. Lever, Z. Ikonić, and R. W. Kelsall, "Extended density-matrix model applied to silicon-based terahertz quantum cascade lasers," Phys. Rev. B, Condens. Matter, vol. 85, no. 23, 2012, Art. no. 235427. [Online]. Available: http://link.aps.org/doi/ 10.1103/PhysRevB.85.235427

[27] B. A. Burnett and B. S. Williams, "Density matrix model for polarons in a terahertz quantum dot cascade laser," Phys. Rev. B, Condens. Matter, vol. 90, no. 15, 2014, Art. no. 155309. [Online]. Available: http://link.aps.org/doi/10.1103/PhysRevB.90.155309

[28] B. A. Burnett and B. S. Williams, "Origins of terahertz difference frequency susceptibility in midinfrared quantum cascade lasers," Phys. Rev. Appl., vol. 5, no. 3, 2016, Art. no. 034013. [Online]. Available: http://link.aps.org/doi/10.1103/PhysRevApplied.5.034013

[29] A. Grier et al., "Origin of terminal voltage variations due to self-mixing in terahertz frequency quantum cascade lasers," Opt. Exp., vol. 24, no. 19, pp. 21948-21956, 2016. [Online]. Available: http://www.osapublishing.org/abstract.cfm?uri=oe-24-19-21948

[30] A. Yariv, C. Lindsey, and U. Sivan, "Approximate analytic solution for electronic wave functions and energies in coupled quantum wells," $J$. Appl. Phys., vol. 58, no. 9, pp. 3669-3672, 1985. [Online]. Available: http://scitation.aip.org/content/aip/journal/jap/58/9/10.1063/1.335726

[31] G. Bastard, Wave Mechanics Applied to Semiconductor Heterostructures. Les Ulis, France: EDP Sci., 1988.

[32] P. Harrison and A. Valavanis, Quantum Wells, Wires and Dots: Theoretical and Computational Physics of Semiconductor Nanostructures. New York, NY, USA: Wiley, 2016.

[33] I. Savić et al., "Density matrix theory of transport and gain in quantum cascade lasers in a magnetic field," Phys. Rev. B, Condens. Matter, vol. 76, no. 16,2007 , Art. no. 165310.

[34] J. R. Freeman, O. Marshall, H. E. Beere, and D. A. Ritchie, "Improved wall plug efficiency of a $1.9 \mathrm{THz}$ quantum cascade laser by an automated design approach," Appl. Phys. Lett., vol. 93, no. 19, 2008, Art. no. 191119. [Online]. Available: http://scitation.aip.org/content/ aip/journal/apl/93/19/10.1063/1.3030881
[35] V. D. Jovanović et al., "Mechanisms of dynamic range limitations in GaAs/AlGaAs quantum-cascade lasers: Influence of injector doping," Appl. Phys. Lett., vol. 86, no. 21, 2005, Art. no. 211117. [Online] Available: http://scitation.aip.org/content/aip/journal/apl/86/21/10.1063/ 1.1937993

[36] J. D. Cooper, A. Valavanis, Z. Ikonić, P. Harrison, and J. E. Cunningham, "Finite difference method for solving the Schrödinger equation with band nonparabolicity in mid-infrared quantum cascade lasers," J. Appl. Phys., vol. 108, no. 11, 2010, Art. no. 113109. [Online]. Available: http:// scitation.aip.org/content/aip/journal/jap/108/11/10.1063/1.3512981

[37] C. A. Evans, D. Indjin, Z. Ikonić, and P. Harrison, "The role of temperature in quantum-cascade laser waveguides," J. Comput. Electron., vol. 11, no. 1, pp. 137-143, 2012. [Online]. Available: http://dx.doi.org/10.1007/s10825-012-0398-7

[38] C. A. Evans et al., "Thermal modeling of terahertz quantum-cascade lasers: Comparison of optical waveguides," IEEE J. Quantum Electron., vol. 44, no. 7, pp. 680-685, Jul. 2008.

[39] A. Grier et al., "Coherent vertical electron transport and interface roughness effects in AlGaN/GaN intersubband devices," J. Appl. Phys., vol. 118, no. 22, 2015, Art. no. 224308. [Online]. Available: http://dx.doi.org/10.1063/1.4936962

[40] G. Agnew et al., "Efficient prediction of terahertz quantum cascade laser dynamics from steady-state simulations," Appl. Phys. Lett., vol. 106, no. 16, 2015, Art. no. 161105. [Online]. Available: http://scitation. aip.org/content/aip/journal/apl/106/16/10.1063/1.49189 93

[41] D. Indjin, P. Harrison, R. W. Kelsall, and Z. Ikonić, "Mechanisms of temperature performance degradation in terahertz quantum-cascade lasers," Appl. Phys. Lett., vol. 82, no. 9, pp. 1347-1349, 2003. [Online]. Available: http://dx.doi.org/10.1063/1.1558220

Author photographs and biographies not available at the time of publication. 